\title{
Entwicklung des zivilgesellschaftlichen Engagements im Jugend- und jungen Erwachsenenalter
}

\author{
Susanne Kuger (D) - Martina Gille
}

Eingegangen: 16. März 2020 / Überarbeitet: 8. September 2020 / Angenommen: 8. Oktober 2020 / Online publiziert: 10 . November 2020

(C) Der/die Autor(en) 2020

Zusammenfassung Politische Involviertheit und aktive Beteiligung im Ehrenamt werden beide als zentrale Säulen zivilgesellschaftlichen Engagements betrachtet. Zu beiden Konstrukten liegen differenzielle Befunde für zentrale Personenmerkmale vor, die mehrheitlich auf der Analyse querschnittlicher Daten beruhen, sowie nur wenige Belege zur Stabilität über die Lebensspanne. Die Jugend und das junge Erwachsenenalter werden als kritische Phasen für die Entwicklung und Aufrechterhaltung beider Konstrukte angesehen, obwohl bislang nur für Teilabschnitte empirische Belege vorliegen und keine Studien zur Untersuchung des komplexen gegenseitigen Bedingungsgefüges. Sowohl die (differenzielle) Stabilität als auch die wechselseitige Abhängigkeit der beiden Konstruktbereiche im Entwicklungsverlauf von der frühen Jugend bis ins Erwachsenenalter hinein werden anhand der AID:A Daten untersucht. Die Ergebnisse von Strukturgleichungsmodellen belegen unterschiedliche, auch nicht-lineare Veränderungsprozesse für drei Teilbereiche, das Interesse an Politik, die Wichtigkeit des Lebensbereichs Politik und das ehrenamtliche Engagement. Das Geschlecht, das Alter und die Bildung der Personen sowie ausgewählte allgemeine Wertorientierungen stellen sich als relevante Kovariaten in den latenten Wachstumskurvenmodellen und dem autoregressiven Modell heraus.

Schlüsselwörter Politisches Interesse · Vorpolitische Einstellungen · Ehrenamtliches Engagement

PD Dr. S. Kuger $(\varangle) \cdot$ M. Gille

Deutsches Jugendinstitut (DJI), Nockherstraße 2, 81541 München, Deutschland

E-Mail: kuger@dji.de

M. Gille

E-Mail: gille@dji.de 


\title{
The development of civil engagement in adolescents and young adults
}

\begin{abstract}
Political involvement and volunteer work are both regarded as central to civil engagement. Both are associated with person characteristics, but relevant findings are mostly based on the analysis of cross-sectional data. Furthermore, there is only little evidence of stability over the life span. Adolescence and young adulthood are considered to be critical for the development and maintenance of both constructs, although empirical evidence is so far available only for shorter timespans. There are no studies available investigating the complex mutual conditionality. Both, the (differential) stability and the interdependence of the two construct areas during intraindividual development from early adolescence to adulthood are investigated using the AID:A data. The results of structural equation models prove different, partially nonlinear processes of change for three facets: the interest in politics, the subjective importance of politics and volunteer work. The persons' gender, age and level of education as well as selected general value orientations are relevant covariates in the latent growth curve models and an autoregressive model.
\end{abstract}

Keywords Early political orientations · Interest in politics · Volunteer work

Seit Beginn des Jahres 2019 hat das Engagement junger Leute im Kontext der Fridays-for-Future-Bewegung weltweit starke mediale Aufmerksamkeit erfahren. Dabei erweisen sich in einigen Ländern, wie z. B. in Deutschland, insbesondere die jüngeren Jugendlichen, also Schülerinnen und Schüler, und die jungen Frauen als eine besonders aktive Gruppe (vgl. Sommer et al. 2019). Als junge und weltweit expandierende soziale Bewegung liefert die Fridays-for-Future-Bewegung ein eindrucksvolles Beispiel für die politische Mobilisierbarkeit und hohe Engagementbereitschaft junger Leute. Diese wachsende Bedeutung der Klima-Bewegung wird auch in Zusammenhang gebracht mit der möglicherweise generellen Tendenz eines steigenden politischen Interesses vor allem in der jungen Generation (Schneekloth und Albert 2019). Der Anstieg dieser protestorientierten Beteiligung im Rahmen der Fridays-for-Future-Bewegung ist vor dem Hintergrund einer möglichen wechselseitigen Beeinflussung von verschiedenen zivilgesellschaftlichen Beteiligungsformaten auch für die Engagementforschung interessant.

Politischer Protest, konventionelles politisches Engagement wie die Mitarbeit in Parteien auf der einen Seite und das Volunteering („Ehrenamtlichkeit“) sowie die Mitarbeit in Initiativen bzw. Organisationen mit gemeinnützigem Charakter auf der anderen, stellen wesentliche Bereiche des zivilgesellschaftlichen Engagements dar (Blinkert und Klie 2015). Allerdings existieren bisher nur wenige Untersuchungen, die dem Zusammenhang dieser beiden Konstruktbereiche, politischer Partizipation und freiwilligen Engagements, nachgehen (Deutscher Bundestag 2017b). Ausnahmen stellen die AID:A 2014-Studie (Gaiser und Gille 2012; Gille et al. 2014) sowie die internationale Vergleichsstudie von Gabriel (2014 $)$ dar. In beiden Untersuchun-

\footnotetext{
1 Gabriel, O.W. (2014). Politische Beteiligung: Die Qualität der Demokratie und das Konzept guter Staatsbürgerschaft (Manuskript im Auftrag der Bertelsmann-Stiftung, unveröffentlicht).
} 
gen kann nachgewiesen werden, dass politisches Interesse und freiwilliges Engagement sowie auch Beteiligungserfahrungen im politischen Raum und freiwilliges Engagement positiv zusammenhängen. Diese Verknüpfungen beruhen allerdings auf Analysen querschnittlicher Daten und sind daher in ihrer Aussagekraft begrenzt. Bis auf wenige Ausnahmen gibt es kaum Studien, die in einem Längsschnittdesign die Entwicklung politischer Einstellungen und zivilgesellschaftlicher Beteiligung untersuchen (Gille und Kuger im Druck). Gänzlich fehlen Studien, die wechselseitige Beziehungen zwischen den beiden Konstrukten im Verlauf individueller Entwicklungen betrachten. Auch wenn das Jugend- und junge Erwachsenenalter nicht ausschließlich als die prägende Phase für die politische Sozialisation angesehen wird, so hat dieser Lebensabschnitt doch eine relevante Bedeutung (Rippl 2015) und es gilt zu klären, inwieweit sich politisches Interesse und zivilgesellschaftliche Beteiligung in dieser Lebensspanne entwickeln und möglicherweise stabilisieren.

Der vorliegende empirische Beitrag setzt an diesem Forschungsdefizit an und untersucht anhand des AID:A Panels III den Wirkungszusammenhang von politischer Involvierung und ehrenamtlichem Engagement in einem Längsschnittdesign in der Lebensspanne des Jugend- und jungen Erwachsenenalters. Dabei stehen zwei Fragestellungen im Fokus: Zum einen wird untersucht, inwiefern politische Involvierung und zivilgesellschaftliches Engagement stabile Merkmale einer Person während ihres Jugend- und jungen Erwachsenenalters sind. Zum anderen untersucht der Beitrag in welchem Ausmaß politische Involvierung und zivilgesellschaftliches Engagement sich während der Jugend und des jungen Erwachsenenalters gegenseitig bedingen; ob es gegenseitige Einflüsse gibt und ob diese zu ähnlichen Zeitpunkten einsetzen: Führt zivilgesellschaftliches Engagement zu einer stärkeren politischen Involvierung bei jungen Leuten oder gibt es eine umgekehrte Kette zusammenhängender Veränderungen?

\section{Zivilgesellschaftliches Engagement}

Zivilgesellschaftliches Engagement in einem weiten Engagementverständnis konzentriert sich ,nicht nur auf die im Freiwilligensurvey ausschließlich berücksichtigten Formen freiwilligen Engagements als praktisches Tun für gemeinwohlbezogene Belange“", sondern bezieht auch die politische Beteiligung mit ein (Blinkert und Klie 2015). Im Folgenden wird dieses breite Verständnis zunächst theoretisch eingebettet und dargestellt, wie sich die beiden Konzepte der politischen Involvierung und des ehrenamtlichen Engagements dazu verhalten, um anschließend die Forschungsfragen abzuleiten.

\subsection{Politische Involvierung}

Demokratische Gesellschaften sind auf die Beteiligung ihrer Bürgerinnen und Bürger angewiesen. Die politische Kulturforschung verweist darauf, dass eine breite Unterstützung der demokratischen Regierungsform und eine politisch involvierte Bürgerschaft zentrale Voraussetzung für eine lebendige Demokratie sind (Almond und Verba 1963). Die politische Involvierung, die in Umfragestudien häufig über die 
Frage nach dem politischen Interesse operationalisiert wird, hat einen entscheidenden Einfluss darauf, ob und in welchem Ausmaß sich Befragte politisch beteiligen. Empirisch bestätigt hat dies beispielsweise Gabriel (2013) anhand von ALLBUSDaten unter Anwendung des „Civic Voluntarism Model“. Nach diesem Erklärungsmodell für politische Partizipation von Verba et al. (1995) ist politische Beteiligung von drei Faktorbereichen abhängig: von Ressourcen und Kompetenzen, von Motivationen und Interessen und schließlich vom Eingebundensein in soziale Netzwerke. Nach den Befunden von Gabriel (2013) ist politisches Interesse der wichtigste Erklärungsfaktor für politische Partizipation neben weiteren nachgeordneten Faktoren wie dem sozioökonomischen Status, dem Migrationshintergrund und der Mitgliedschaft in Freiwilligenorganisationen. Auch Ergebnisse des DJI-Surveys AID:A 2009 weisen auf die Bedeutung des politischen Interesses für die aktive Beteiligung an der Gestaltung der Zivilgesellschaft und die Übernahme freiwilliger Aufgaben (,eines Amtes oder einer Funktion“) in Vereinen und Verbänden hin (Gille et al. 2014).

Neben dem politischen Interesse ist auch die subjektive Bewertung des Lebensbereichs Politik ein weiterer zentraler Indikator für politische Involvierung. Wenn man junge Menschen nach dem Stellenwert von Politik in ihrem Leben fragt und dies mit anderen Bereichen wie Herkunftsfamilie, Freunden, Freizeit und Arbeit vergleicht, so erhält Politik einen relativ geringen Stellenwert (vgl. Gille 2016).

Sowohl das politische Interesse als auch die Wichtigkeit des Lebensbereichs Politik sind eng mit verschiedenen Personenmerkmalen assoziiert. Aus wiederholten querschnittlichen Studien mit Zeitreihendaten wurde in den letzten Jahren die Schlussfolgerung steigenden politischen Interesses bei Jugendlichen abgeleitet, wie es auch im Zusammenhang mit der Fridays-for-future Bewegung diskutiert wird (Deutscher Bundestag 2017a; Gille 2018; Schneekloth und Albert 2019). Intra-individuelle Entwicklungen lassen sich aufgrund spärlich vorhandener Daten nur bedingt untersuchen. Mittels Daten der BIJU-Studie konnten Baumert et al. (2016) nachweisen, dass in der Altersspanne zwischen 14 und 17 Jahren sowie im jungen Erwachsenenalter jeweils ein substantieller Anstieg des politischen Interesses stattgefunden hat. Männer und Frauen zeigen dabei zwar nahezu parallele Entwicklungen, jedoch mit deutlichen Niveauunterschieden, die über die Zeit eher zunehmen. Auch die Ergebnisse von Grob (2006) lassen den Schluss zu, dass schon die frühe und mittlere Adoleszenz - hier bezogen auf die Altersspanne von 12 bis 16 Jahren - besonders wichtig für die Entwicklung des politischen Interesses sind.

Weiterhin besonders wichtig ist das Geschlecht der befragten Personen: Mädchen und junge Frauen sind weniger an Politik interessiert bzw. geben dem Lebensbereich Politik einen geringeren Stellenwert als die männliche Vergleichsgruppe (z. B. Gille und Kuger im Druck). Schließlich wird Bildung als wichtiger Einflussfaktor für politische Involvierung diskutiert. Dies wird allerdings an einem insgesamt gestiegenen Interesse an Politik parallel zur Bildungsexpansion der letzten Jahrzehnte festgemacht (Hadjar und Becker 2006).

\subsection{Ehrenamtliches Engagement}

Neben Einstellungen zu Politik ist das freiwillige Einbringen im Ehrenamt eine wichtige Komponente des zivilgesellschaftlichen Engagements. Die wichtigsten in- 
stitutionellen Orte für freiwilliges Engagement sind nach wie vor Vereine, wie die Ergebnisse des Freiwilligensurveys 2014 sowie der Shell Jugendstudie 2019 zeigen (Schneekloth und Albert 2019; Simonson und Vogel 2017). Ergebnisse früherer AID:A-Studien ermöglichen diesbezüglich einen vertieften Einblick vor allem in die aktive Übernahme von Ämtern und Funktionen in Vereinen, Verbänden und Organisationen. Der Anteil junger Menschen (18 bis 32 Jahre), die mindestens ein Amt bzw. Funktion in einem Verein übernommen haben, beträgt $24 \%$ bzw. $23 \%$ im AID:ASurvey 2009 bzw. 2014 (Gille 2015). Die Aktivitäten in Sportvereinen stellen dabei die wichtigste Form von Vereinsbeteiligung in dieser Altersspanne dar, wobei sich junge Männer stärker einbringen als junge Frauen (ebenda). Kirchliche und religiöse Gruppen spielen ebenso wie Gesangs- und Musikvereine sowie Theatergruppen die zweitwichtigste Rolle für junge Menschen, wobei diese Aktivitäten stärker auf das Interesse bei Mädchen und jungen Frauen stoßen. Gewerkschaften, die Freiwillige Feuerwehr und Heimatvereine spielen ebenso wie die politischen Organisationen und Parteien nur eine nachgeordnete Rolle sowohl bei jungen Frauen als auch bei den jungen Männern. Der Freiwilligensurvey 2014 erfasst zivilgesellschaftliches Engagement im Vergleich zu AID:A in einem umfassenderen Sinne, d.h. als unentgeltliches, gemeinwohlorientiertes und über das reine mitmachen hinausgehende Engagement, wobei die Orte des Engagements nicht nur auf Vereine beschränkt sind. $47 \%$ der befragten 14- bis 29-Jährigen gaben an, in den letzten 12 Monaten freiwillig engagiert zu sein, die jungen Männer mit 49\% in einem etwas höheren Ausmaß als die jungen Frauen mit $45 \%$ (Vogel et al. 2017). Für die Wahl der Tätigkeitsfelder junger Frauen und Männer erhält man ein ganz ähnliches Geschlechterprofil wie bei dem zuvor dargestellten Vereinsengagement. Darüber hinaus zeigen sich sowohl in AID:A 2014 wie auch im Freiwilligensurvey 2014 vergleichbare Selektionseffekte in der Ausübung freiwilligen Engagements im Hinblick auf Lebensalter, Bildung und Migrationshintergrund. Ältere, geringer Gebildete und junge Leute mit Migrationshintergrund sind weniger häufig freiwillig engagiert als Jüngere, höher Gebildete und junge Menschen mit Migrationshintergrund (vgl. Gille 2015; Kausmann et al. 2017).

In der Engagementforschung stellen sowohl die Frage nach der Verknüpfung von politischer Partizipation und freiwilligem Engagement als auch die Untersuchung von Engagementkarrieren im biographischen Verlauf häufig benannte Forschungsdesiderata dar (vgl. Deutscher Bundestag 2017a, 2017b). Nur wenige Studien verfügen über ausreichend Daten zur Untersuchung dieser Fragen. Eine der wenigen Ausnahmen stellt die Messwiederholungstudie von Reinders (2014) dar, in der Schülerinnen und Schüler im Alter von 14 Jahren und nach einem Jahr im Alter von 15 Jahren nochmals zu ihrem gemeinnützigen und politischen Engagement befragt worden sind. Die Ergebnisse zeigen, dass die Erfahrung von gemeinnützigen Tätigkeiten sowohl die politischen Aktivitäten als auch die politische Beteiligungsbereitschaft bei den Befragten erhöhte. Die Befunde einer drei Erhebungswellen umfassenden Panelstudie bei Schülerinnen und Schülern von Dahl und Abdelzadeh (2017) bestätigen keine gerichteten, längsschnittlichen Zusammenhänge von zivilgesellschaftlichem Engagement auf (erhöhtes) politisches Interesse. 


\section{Beitrag der vorliegenden Studie}

Die Literatursichtung zeigt, dass politische Einstellungen und das freiwillige Einbringen im Ehrenamt wichtige Komponenten des zivilgesellschaftlichen Engagements darstellen, welches als Grundvoraussetzung für demokratische Gesellschaften gilt. Zugleich können deutliche Forschungsdesiderata hinsichtlich zweier Forschungsrichtungen identifiziert werden. Zum einen bestehen Unklarheiten hinsichtlich der Stabilität oder Veränderbarkeit der beiden Konstruktbereiche während der Jugend und jungen Adoleszenz, zum anderen finden sich widersprüchliche Befunde hinsichtlich der wechselseitigen Beziehung der beiden Konstruktbereiche während des Aufwachsens. Diesen beiden Forschungslücken widmet sich dieser Beitrag, indem er die politische Involvierung und das ehrenamtliche Engagement entlang der biografischen Verläufe junger Menschen verknüpft. Die zwei zentralen Fragestellungen des Beitrags sind:

1. Inwieweit sind die Konstrukte a) der politischen Involvierung, wie a1) das politische Interesse und a2) die Wichtigkeit von Politik, sowie b) die ehrenamtliche Beteiligung in Vereinen stabil über den Lebensverlauf? Gemäß des oben dargelegten Forschungsstands wird von einer Zunahme der politischen Involviertheit ausgegangen. Allerdings können aufgrund der Literatur keine Annahmen über die Form des Wachstums gemacht werden. Zum Verlauf des ehrenamtlichen Engagements können aufgrund der Literatur keinerlei Wachstumshypothesen aufgestellt werden. Weiterhin geht der Beitrag davon aus, dass verschiedene Personenmerkmale, allen voran das Geschlecht und das Bildungsniveau der Personen, mit dem Ausprägungsniveau der drei Konstrukte zusammenhängen. Es können jedoch keine differenzierten Annahmen über Zusammenhänge zwischen Personenmerkmalen und den Veränderungsprozessen aufgestellt werden.

2. Gibt es wechselseitige Zusammenhänge der beiden Konstruktbereiche während der intra-individuellen Entwicklung in der Jugend und jungen Adoleszenz? Im Detail wird untersucht, ob das zivilgesellschaftliche Engagement einer Person während des individuellen Entwicklungsverlaufs ihre politische Involvierung verstärkt oder abschwächt, bzw. ob sich umgekehrte gerichtete Beziehungsmuster erkennen lassen. Aufgrund der heterogenen Literaturlage können für diese Forschungsfrage keine gerichteten Hypothesen aufgestellt werden,

\section{Studie und methodisches Vorgehen}

In der vorliegenden Studie wird zurückgegriffen auf Daten der DJI-AID:A Studien. AID:A stellt den konzeptionellen Rahmen einer Reihe empirischer Surveystudien dar, die in ihrem Kern das Aufwachsen in Deutschland in den Alltagswelten der Kinder, Jugendlichen und jungen Erwachsenen untersuchen. In den Beitrag gehen Daten der Studien AID:A I (Erhebung 2009), AID:A II (Erhebung 2014) und des AID:A Panels III (Erhebung 2018) ein. 


\subsection{Befragungsvorgehen und Instrumente}

In den ersten drei Erhebungswellen AID:A I, II und Panel III wurden mittels Telefoninterviews Informationen u. a. zu den Lebensumständen, Bildungskarrieren, Einstellungen, Paarbeziehungen und Zukunftsplänen von Kindern, Jugendlichen, jungen Erwachsenen und Familien in Deutschland erfasst. In dieser Vielfalt von Themen wurden Jugendliche ab dem Alter von 13 (AID:A I), 12 (AID:A II) bzw. 12 Jahren (AID:A Panel III) auch zu ihren politischen Einstellungen und ihrem ehrenamtlichen Engagement befragt, genauer zu ihrem Interesse an Politik (IntPolitik), der Wichtigkeit des Lebensbereiches Politik (WichtigPolitik) sowie genauer zu der Art und dem Umfang ihrer Beteiligung und ihrem Einbringen in ehrenamtliche Tätigkeiten (kurz auch „Ehrenamt“). Die Fragenformulierung über die drei Erhebungszeitpunkte blieb identisch. Allerdings wurde zum dritten Erhebungszeitpunkt (AID:A Panel III 2018) retrospektiv zusätzliche Information über das ehrenamtliche Engagement der Befragten erhoben (,ZEngagement)“. Ihr politisches Interesse bekannten die Personen zu der Einzelfrage „Wie stark interessieren Sie sich für Politik?“ auf einer fünfstufigen Skala von $1=$ überhaupt nicht, $2=$ wenig, $3=$ mittel, $4=$ stark bis $5=$ sehr stark. Die Wichtigkeit des Lebensbereichs Politik wurde eingebettet in eine längere Liste von Lebensbereichen (darunter z.B. auch Familie, Freunde, Freizeit, Schule, Kunst und Kultur sowie Gesundheit) auf einer sechsstufigen Skala von $1=$ überhaupt nicht wichtig bis $6=$ sehr wichtig abgefragt. Das ehrenamtliche Engagement wurde in allen drei Wellen im Nachgang zur Frage nach der Beteiligung in einem breiten Spektrum von Vereinen oder Gruppen erfasst. Entlang der angewandten Liste von Vereinen und Organisationen wurden die jeweils dort aktiven jungen Leute danach gefragt, ob sie ein Amt oder eine Funktion ausüben. Somit konnte auch ein mehrfaches ehrenamtliches Engagement erfasst werden. Im AID:A Panel III 2018 wurde darüber hinaus erfasst, ob Befragte noch eine zusätzliche weitere ehrenamtliche Aufgabe in Projekten oder Initiativen übernommen haben, die außerhalb der abgefragten Liste von Vereinen und Organisation stattfinden.

Als in der Literatur bedeutend für die Entwicklung politischer Einstellungen und des ehrenamtlichen Engagements diskutierte Personenmerkmale werden einbezogen: das Geschlecht der Studienteilnehmer und -teilnehmerinnen (1 = männlich; $2=$ weiblich $)$, ihr Alter, ihr Migrationshintergrund ( $1=$ liegt nicht vor, $2=$ mind. ein Elternteil oder Person selbst im Ausland geboren), ihr Bildungsabschluss bzw. der Bildungsabschluss, der mit dem von ihr zum Zeitpunkt der Befragung besuchten Schulzweig üblicherweise erreicht wird.

Zur Approximation möglicher Motivationen der befragten Personen sich zivilgesellschaftlich zu engagieren, wurden darüber hinaus als zweites Prädiktorenset Angaben der Befragten zu vier ausgewählten allgemeinen Wertorientierungen aufgenommen. Die vier Angaben repräsentieren jeweils Wertebereiche, die sich aus der Wertetheorie von Helmut Klages (1993) ableiten lassen. Die Befragten wurden gebeten, jede angeführte Verhaltensweise danach zu bewerten, ,wie wichtig es ihnen persönlich ist, so zu sein oder sich so zu verhalten" ( 1 = überhaupt nicht wichtig bis $6=$ sehr wichtig) sind: „Pflichtbewusstsein“, „Rücksicht auf andere nehmen“, „Kritisch sein“ und „Tun und lassen was ich will“. Pflichtbewusstsein steht im Kontext von Akzeptanz, Materialismus und Leistungsbereitschaft, „Rücksicht auf andere 
nehmen“ repräsentiert prosoziales Verhalten, „Kritisch sein“ steht in Zusammenhang mit Selbstbehauptung und der Verwirklichung eigener Fähigkeiten und „Tun und lassen" verweist auf eine eher hedonistische Orientierung, die üblicherweise eher für jüngere Altersgruppen wichtig ist. In die Analyse werden Mittelwerte der wiederholt abgefragten Wertvorstellungen aufgenommen.

\subsection{Stichprobe}

Die beiden Studien AID:A I und AID:A II befragten jeweils für Deutschland repräsentative Einzelstichproben. Jugendliche gaben dabei zu sich selbst Auskunft, für Kinder bis zum Alter von 12 (AID:A I) bzw. 11 Jahren (AID:A II) wurde ein Elternteil als Auskunftsperson stellvertretend um die Beantwortung des Fragebogeninstruments gebeten (für eine ausführliche Studienbeschreibung der AID:A Studien s. auch https://surveys.dji.de). Ausgehend von der Erhebung AID:A I in 2009 wurde ein Teil der Befragten 2014 in AID:A II und 2018 für das AID:A Panel III wiederholt kontaktiert. Aufgrund des komplexen Designs der Verschränkung der AID:A Stichproben (vgl. auch Walper et al. 2015) liegen aus den drei Wellen unterschiedlich viele gültige Antworten vor. Zur Minimierung der fehlenden Werte in Einzelwellen wurden nur Personen in die Analyse aufgenommen, die mindestens zum zweiten und dritten Messzeitpunkt Angaben zu ihren politischen Einstellungen und ihrem ehrenamtlichen Engagement gemacht hatten $(n=1385){ }^{2}$ Tab. 1 fasst die Stichprobenmerkmale der für die Hauptspezifikation des Beitrags genutzten Teilstichprobe zusammen.

Die Stichprobenbeschreibung weist auf zweierlei Schieflagen hin: Zum einen sind Personen mit höherer Bildung oder besserem Bildungshintergrund überrepräsentiert. Dabei muss berücksichtigt werden, dass die Angabe den zum letzten Messzeitpunkt

Tab. 1 Merkmale der ausgewählten AID:A Stichprobe

\begin{tabular}{|c|c|}
\hline$N$ & 1385 \\
\hline Alter in Jahren zu t 1 AID:A I: M; SD (Range) & 11,$8 ; 3,3$ (6-19 Jahre) \\
\hline Alter in Jahren zu t2 AID:A II: M; SD (Range) & 16,$8 ; 3,3$ (12-24 Jahre) \\
\hline Alter in Jahren zu t 3 AID:A Panel III: M; SD (Range) & 20,$3 ; 3,3$ (15-28 Jahre) \\
\hline Anteil weiblicher Befragter & $47,9 \%$ \\
\hline $\begin{array}{l}\text { Migrationshintergrund (mind. ein Elternteil oder Person selbst } \\
\text { nicht in Deutschland geboren) }\end{array}$ & $10,8 \%$ \\
\hline HISEI der Eltern im Haushalt Md (Range) & $57(16-90)$ \\
\hline \multicolumn{2}{|l|}{$\begin{array}{l}\text { Eigener Bildungsstatus zu AID:A Panel III } 2018 \\
\text { (schon erworben oder in der aktuellen Schulausbildung angestrebt) }\end{array}$} \\
\hline Kein Abschluss oder Hauptschulabschluss & $2,5 \%$ \\
\hline Mittlerer Schulabschluss & $14,3 \%$ \\
\hline (Fach-)Hochschulreife & $83,1 \%$ \\
\hline
\end{tabular}

Quelle: DJI-Surveys AID:A I (2009), AID:A II (2014) und AID:A Panel III (2018); eigene Berechnungen, ungewichtet

\footnotetext{
2 Alle Analysen wurden zudem repliziert für $n=511$ Fälle, für die jeweils alle drei Angaben zu allen drei Messzeitpunkten vorliegen. Die Ergebnismuster zeigten keine bedeutenden Unterschiede zur hier dargestellten Variante.
} 
erreichten Bildungsabschluss wiedergibt und damit Nachqualifikationen miterfasst sind. Zum anderen ist ersichtlich, dass es nicht ausreichend gut gelungen ist Personen mit Migrationshintergrund zur Teilnahme zu motivieren; sie bleiben etwas unterrepräsentiert. Trotz dieser Probleme eignet sich diese Stichprobe sehr gut zur Analyse von Entwicklungsverläufen der politischen Einstellungen von Jugendlichen und jungen Erwachsenen. Die Befragten dieser Längsschnittkohorte waren zum ersten Messzeitpunkt zwischen 6 und 19 Jahren alt und 2018 zwischen 15 und 28 Jahren alt. Ihr individueller Entwicklungsverlauf lässt sich über die wichtige Zeitspanne der Jugend und des jungen Erwachsenenalters mit all seinen Brüchen und Übergängen verfolgen unter Kontrolle von Bildungsniveau und Migrationshintergrund.

\subsection{Vorgehen}

In den Auswertungen kommen korrelative Verfahren, speziell Modellierungen von Strukturgleichungsmodellen zur Anwendung, um die eher konstruktzentrierte Forschungsfrage 1, als auch die Frage $2 \mathrm{zu}$ individuellen Entwicklungsverläufen zu beantworten. Im ersten Schritt wird die Konstruktstabilität von Intersse an Politik, Wichtigkeit von Politik und Ehrenamt sowie die Abhängigkeit der Stabilität von anderen Personenmerkmalen analysiert. Ein stärkerer Fokus auf die Entwicklungen der Personen im untersuchten 10-Jahres Zeitraum wird im zweiten Abschnitt gelegt, der die längsschnittlichen gegenseitigen Abhängigkeiten der Merkmale voneinander untersucht.

Die Stabilität der beiden untersuchten Einstellungen sowie des Ehrenamts der Befragten wurden mittels unkonditionierter latenter Wachstumskurvenmodelle untersucht, in denen die Ausgangs- bzw. Grundausprägung (latente Variable Intercept $i$ ) eines Merkmals getrennt von der Veränderung des Merkmals über die Zeit (latente Variable Slope $s$ ) modelliert wird (Modell 1). Über die drei Zeitpunkte werden lineare Veränderungen angenommen sowie explorativ non-lineare Veränderungen geprüft. Zusätzlich aufgenommene Kovariablen geben in jeweils einem weiteren Modell 2 Auskunft über Beziehungen von Personenmerkmalen zum Ausgangsstand und zu den Veränderungen in den drei zentralen Untersuchungsmerkmalen.

Schließlich werden die gegenseitigen Abhängigkeiten der Merkmale im Zeitverlauf mittels eines autoregressiven kreuzverzögerten Modells modelliert. Für diese Modellierung werden die beiden untersuchten Merkmale für politische Einstellungen Interesse an Politik und Wichtigkeit Politik pro Messzeitpunkt zu einem latenten Faktor zusammengefasst. Das Ehrenamt der Studienteilnehmer und -teilnehmerinnen wird zu den beiden Erhebungszeitpunkten 1 (AID:A I 2009) und 2 (AID:A II 2014) manifest modelliert und für den dritten Erhebungszeitpunkt aus der Frage nach der Anzahl der aktuellen ehrenamtlich ausgeführten Funktionen in der Liste vorgegebener Vereine und Verbände sowie dem zusätzlichem Engagement jenseits der vorgegebenen Liste latent zusammengefasst. Im Modell werden insgesamt vier Modellmerkmalsgruppen modelliert: 1) die Stabilität der Konstrukte als Regressionskoeffizienten eines späteren Zeitpunkts $\left(t_{x}\right)$ auf den jeweils vorhergehenden Zeitpunkt $\left(t_{x}-1\right)$; 2) Korrelationen zwischen den beiden Konstruktbereichen zu den jeweiligen Erhebungszeitpunkten; 3) die gegenseitigen Beziehungen zwischen den politischen Einstellungen und dem ehrenamtlichen Engagement durch zeitverzö- 


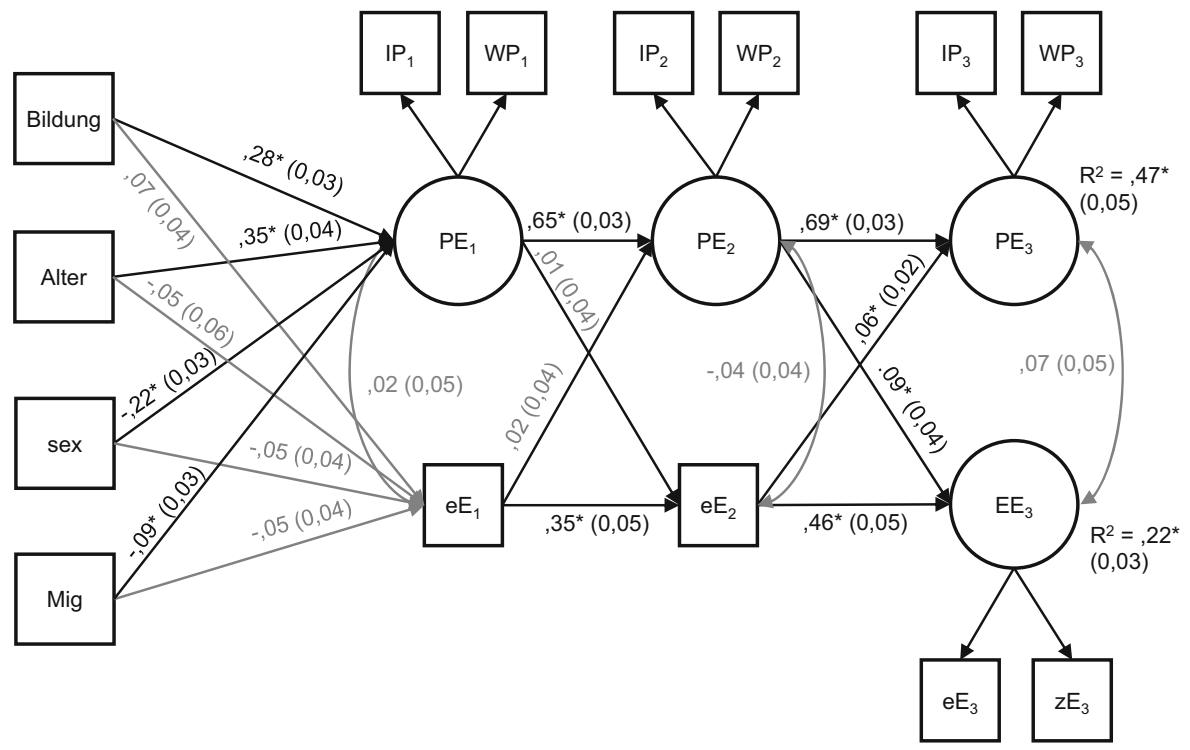

Abb. 1 Kreuzklassifiziertes Modell der gegenseitigen Beeinflussung politischer Einstellungen und des ehrenamtlichen Engagements. (Angetragen sind standardisierte Regressionskoeffizienten (sowie Standardfehler des Schätzers). Nicht signifikante Pfade sind grau dargestellt. Faktorladungen sind der Lesbarkeit halber nicht angetragen, jedoch durchweg signifikant. IntPolitik Interesse an Politik, WichtigPolitik Wichtigkeit des Lebensbereichs Politik, ZEngagement zusätzliches Engagement. Quelle: DJI-Surveys AID:A I (2009), AID:A II (2014) und AID:A Panel III (2018); eigene Berechnungen ungewichtet)

gerte Regressionskoeffizienten eines Konstrukts $x$ zu einem Zeitpunkt $\left(t_{x}\right)$ auf das andere Konstrukt $y$ zum vorhergehenden Erhebungszeitpunkt $\left(t_{y}-1\right)$; 4) Zusammenhänge zentraler Personenmerkmale mit den Einstellungen und dem Engagement zum ersten Erhebungszeitpunkt. Abb. 1 skizziert das resultierende Modell mit allen untersuchten Beziehungspfaden.

Die Analysen zur Stichprobendeskription wurden in IBM SPSS 22 durchgeführt, alle weiteren Modelle wurden in MPlus 7.1 unter Hinzuziehung des Full Information Maximum Likelihood (FIML) Schätzverfahrens modelliert. Die Ergebnisse berichten nicht gewichtete Stichprobenwerte und standardisierte Regressionskoeffizienten.

\section{Ergebnisse}

Die Reihenfolge der Darstellung der Ergebnisse im Ergebnisbericht orientiert sich an der der Forschungsfragen. Deskriptive Ergebnisse der beiden politischen Einstellungen Interesse an Politik und Wichtigkeit Politik sowie des ehrenamtlichen Engagements sind in Tab. 2 angetragen. Danach werden die unkonditionierten Wachstumskurvenmodelle zur Beschreibung der Stabilität der drei Konstrukte dargestellt (Abschn. 4.1; Modelle 1 in Tab. 3). Abschn. 4.1 beschreibt auch Zusammenhänge der Grundausprägung der Personen sowie der Zeitveränderungen mit den ausgewählten Personenmerkmalen (Modelle 2 in Tab. 3). Im Abschn. 4.2 werden die Ergebnisse 
Tab. 2 Deskriptive Ergebnisse der untersuchten Einstellungen und Funktionsübernahmen

\begin{tabular}{|c|c|c|c|}
\hline & $N$ & M & SD \\
\hline \multicolumn{4}{|c|}{ Interesse an Politik ( $1=$ überhaupt nicht bis $5=$ sehr stark $)$} \\
\hline AID:A I 2009 & 511 & 2,96 & 1,02 \\
\hline AID:A II 2014 & 1313 & 3,10 & 0,99 \\
\hline Panel III 2018 & 1385 & 3,18 & 1,09 \\
\hline \multicolumn{4}{|c|}{ Wichtigkeit des Lebensbereichs Politik ( $1=$ überhaupt nicht wichtig bis $6=$ sehr wichtig) } \\
\hline AID:A I 2009 & 511 & 3,89 & 1,16 \\
\hline AID:A II 2014 & 1309 & 3,78 & 1,11 \\
\hline Panel III 2018 & 1385 & 4,02 & 1,06 \\
\hline \multicolumn{4}{|c|}{ Anzahl Funktionen im ehrenamtlichen Engagement } \\
\hline AID:A I 2009 & 511 & 0,39 & 0,63 \\
\hline AID:A II 2014 & 1314 & 0,39 & 0,68 \\
\hline Panel III 2018 & 1385 & 0,34 & 0,59 \\
\hline \multicolumn{4}{|c|}{ Retrospektive Erfassung zusätzlichen ehrenamtlichen Engagements } \\
\hline $\begin{array}{l}\text { Zusätzliches Engage- } \\
\text { ment }\end{array}$ & 1385 & 0,37 & 0,48 \\
\hline
\end{tabular}

Quelle: DJI-Surveys AID:A I (2009), AID:A II (2014) und AID:A Panel III (2018); eigene Berechnungen, ungewichtet

der gemeinsamen Betrachtung gegenseitiger längsschnittlicher Abhängigkeiten der Verläufe berichtet.

Ein Blick auf die Verteilungen der interessierenden Merkmale Interesse an Politik, Wichtigkeit Politik und Ehrenamt zu den drei Messzeitpunkten ist durch die deskriptiven Ergebnisse in Tab. 2 möglich.

Die Datenlage weist anhand der ungewichteten Stichprobenwerte zufriedenstellende Variabilität der Daten auf. Insgesamt geben die Befragten an, durchschnittlich stark an Politik interessiert zu sein und den Lebensbereich Politik als eher wichtig anzusehen. Durchschnittlich übernahm etwa jede dritte Person eine aktive Funktion in einem Ehrenamt.

\subsection{Grundausprägung und Stabilität der Einstellungen und des Engagements}

Die Stabilität der drei interessierenden Merkmale Interesse an Politik, Wichtigkeit Politik und Ehrenamt sowie mögliche Einflussfaktoren wurde mittels latenter Wachstumskurvenmodelle untersucht. Die Ergebnisse sind in Tab. 3 angetragen. Die Modelle wurden unter der Annahme linearer Veränderungen geschätzt, wobei die unkonditionierten latenten Wachstumskurvenmodelle eine insgesamt gute Anpassung der Daten an das lineare Modell für Interesse an Politik und Ehrenamt zeigen (jeweils Modell 1 in Tab. 3). Das Interesse an Politik in der untersuchten Stichprobe startet auf einem mittleren Niveau ( $M=2,92$ auf einer Skala von 1-5) und nimmt im Verlauf der Zeit um 0,14 Skalenpunkte pro Erhebungswelle zu. Sowohl der Ausgangswert als auch die Veränderung zeigen signifikante Varianz in der Stichprobe. Die Veränderung hängt dabei nicht mit dem Ausgangswert der Personen zusammen.

Die Linearitätsannahme für die Veränderung des Modells für Wichtigkeit Politik führt zu unzureichendem Modell-Fit (vgl. Modell 1 zur Wichtigkeit Politik in 
Tab. 3 Latente Wachstumskurvenmodelle für Interesse an Politik, die Wichtigkeit des Lebensbereichs Politik und ehrenamtliches Engagement ohne (Modell 1) und mit (Modell 2) Kovariablen

\begin{tabular}{|c|c|c|c|c|c|c|}
\hline & \multicolumn{2}{|c|}{ Interesse an Politik } & \multicolumn{2}{|c|}{ Wichtigkeit Politik } & \multicolumn{2}{|l|}{ Ehrenamt } \\
\hline & $\begin{array}{l}\text { Modell } 1 \\
\text { (Koeff; } \\
\text { SE) }\end{array}$ & $\begin{array}{l}\text { Modell } 2 \\
\text { (Koeff; } \\
\text { SE) }\end{array}$ & $\begin{array}{l}\text { Modell } 1 \\
\text { (Koeff; } \\
\text { SE) }\end{array}$ & $\begin{array}{l}\text { Modell } 2 \\
\text { (Koeff; } \\
\text { SE) }\end{array}$ & $\begin{array}{l}\text { Modell } 1 \\
\text { (Koeff; } \\
\text { SE) }\end{array}$ & $\begin{array}{l}\text { Modell } 2 \\
\text { (Koeff; } \\
\text { SE) }\end{array}$ \\
\hline \multicolumn{7}{|l|}{ Koeffizienten } \\
\hline \multirow[t]{2}{*}{$\mathrm{i}$} & $\begin{array}{l}2,92 * \\
(0,03)\end{array}$ & - & $\begin{array}{l}3,73 * \\
(0,04)\end{array}$ & - & $\begin{array}{l}\mathbf{0 , 4 2 *} \\
(\mathbf{0 , 0 2})\end{array}$ & - \\
\hline & $\begin{array}{l}\mathbf{0 , 5 3 *} \\
(\mathbf{0 , 0 8})\end{array}$ & - & $\begin{array}{l}0,49 * \\
(0,11)\end{array}$ & - & $\begin{array}{l}\mathbf{0 , 1 7} * \\
(\mathbf{0 , 0 5})\end{array}$ & - \\
\hline \multirow[t]{2}{*}{ Mittelwert } & $\begin{array}{l}0,14 * \\
(0,02)\end{array}$ & - & $\begin{array}{l}\mathbf{0 , 1 4} * \\
(\mathbf{0 , 0 2})\end{array}$ & - & $\begin{array}{l}-0,04 * \\
(0,01)\end{array}$ & - \\
\hline & $\begin{array}{l}0,10 * \\
(0,04)\end{array}$ & - & $\begin{array}{l}\mathbf{0 , 1 3} * \\
(\mathbf{0 , 0 5})\end{array}$ & - & $\begin{array}{l}\mathbf{0 , 0 3} \\
(\mathbf{0 , 0 2})\end{array}$ & - \\
\hline Korrelation i und s & $\begin{array}{l}-0,08 \\
(0,16)\end{array}$ & $\begin{array}{l}0,15 \\
(0,38)\end{array}$ & $\begin{array}{l}-0,34 * \\
(0,17)\end{array}$ & $\begin{array}{l}-0,27 \\
(0,27)\end{array}$ & $\begin{array}{l}-0,49 * \\
(0,18)\end{array}$ & $\begin{array}{l}-0,49 * \\
(0,18)\end{array}$ \\
\hline \multicolumn{7}{|l|}{ Koeffizienten } \\
\hline \multicolumn{7}{|c|}{ Zusammenhang von Intercept mit } \\
\hline Alter & - & $\begin{array}{l}\mathbf{0 , 3 0} * \\
(\mathbf{0 , 0 5})\end{array}$ & - & $\begin{array}{l}0,28 * \\
(0,06)\end{array}$ & - & $\begin{array}{l}-0,08 \\
(0,06)\end{array}$ \\
\hline Geschlecht & - & $\begin{array}{l}-0,19 * \\
(0,05)\end{array}$ & - & $\begin{array}{l}-0,17 * \\
(0,06)\end{array}$ & - & $\begin{array}{l}-0,06 \\
(0,06)\end{array}$ \\
\hline Migrationshintergrund & - & $\begin{array}{l}-0,15 * \\
(0,04)\end{array}$ & - & $\begin{array}{l}-0,02 \\
(0,05)\end{array}$ & - & $\begin{array}{l}-0,07 \\
(0,06)\end{array}$ \\
\hline Schulbildung & - & $\begin{array}{l}\mathbf{0 , 2 3} * \\
(\mathbf{0 , 0 5})\end{array}$ & - & $\begin{array}{l}0,21 * \\
(0,06)\end{array}$ & - & $\begin{array}{l}0,10^{+} \\
(0,06)\end{array}$ \\
\hline $\begin{array}{l}\text { Werteinstellung „Pflicht- } \\
\text { bewusstsein““ }\end{array}$ & - & $\begin{array}{l}-0,06 \\
(0,05)\end{array}$ & - & $\begin{array}{l}0,04 \\
(0,06)\end{array}$ & - & $\begin{array}{l}0,10^{+} \\
(0,06)\end{array}$ \\
\hline $\begin{array}{l}\text { Werteinstellung ,Kri- } \\
\text { tisch sein“ }\end{array}$ & - & $\begin{array}{l}\mathbf{0 , 3 1} * \\
(\mathbf{0 , 0 5})\end{array}$ & - & $\begin{array}{l}\mathbf{0 , 3 8 *} \\
(\mathbf{0 , 0 7})\end{array}$ & - & $\begin{array}{l}0,03 \\
(0,06)\end{array}$ \\
\hline $\begin{array}{l}\text { Werteinstellung „Tun } \\
\text { und lassen was ich will“" }\end{array}$ & - & $\begin{array}{l}-0,01 \\
(0,04)\end{array}$ & - & $\begin{array}{l}-0,01 \\
(0,05)\end{array}$ & - & $\begin{array}{l}-0,01 \\
(0,06)\end{array}$ \\
\hline $\begin{array}{l}\text { Werteinstellung „Rück- } \\
\text { sicht nehmen“ }\end{array}$ & - & $\begin{array}{l}0,06 \\
(0,05)\end{array}$ & - & $\begin{array}{l}0,04 \\
(0,06)\end{array}$ & - & $\begin{array}{l}-0,00 \\
(0,06)\end{array}$ \\
\hline \multicolumn{7}{|c|}{ Zusammenhang von Slope mit } \\
\hline Alter & - & $\begin{array}{l}-0,44 * \\
(0,14)\end{array}$ & - & $\begin{array}{l}-0,45 * \\
(0,12)\end{array}$ & - & $\begin{array}{l}-0,01 \\
(0,09)\end{array}$ \\
\hline Geschlecht & - & $\begin{array}{l}-0,12 \\
(0,08)\end{array}$ & - & $\begin{array}{l}0,05 \\
(0,07)\end{array}$ & - & $\begin{array}{l}-0,02 \\
(0,09)\end{array}$ \\
\hline Migrationshintergrund & - & $\begin{array}{l}0,09 \\
(0,07)\end{array}$ & - & $\begin{array}{l}-0,12 \\
(0,07)\end{array}$ & - & $\begin{array}{l}0,05 \\
(0,08)\end{array}$ \\
\hline Schulbildung & - & $\begin{array}{l}0,08 \\
(0,08)\end{array}$ & - & $\begin{array}{l}-0,04 \\
(0,07)\end{array}$ & - & $\begin{array}{l}-0,16 \\
(0,10)\end{array}$ \\
\hline $\begin{array}{l}\text { Werteinstellung „Pflicht- } \\
\text { bewusstsein“ }\end{array}$ & - & $\begin{array}{l}-0,03 \\
(0,08)\end{array}$ & - & $\begin{array}{l}0,03 \\
(0,07)\end{array}$ & - & $\begin{array}{l}-0,11 \\
(0,09)\end{array}$ \\
\hline $\begin{array}{l}\text { Werteinstellung „Kri- } \\
\text { tisch sein“" }\end{array}$ & - & $\begin{array}{l}\mathbf{0 , 2 2 *} \\
(\mathbf{0 , 1 0})\end{array}$ & - & $\begin{array}{l}0,01 \\
(0,08)\end{array}$ & - & $\begin{array}{l}-0,05 \\
(0,09)\end{array}$ \\
\hline $\begin{array}{l}\text { Werteinstellung „Tun } \\
\text { und lassen was ich will“ }\end{array}$ & - & $\begin{array}{l}-0,05 \\
(0,08)\end{array}$ & - & $\begin{array}{l}-0,00 \\
(0,07)\end{array}$ & - & $\begin{array}{l}-0,08 \\
(0,09)\end{array}$ \\
\hline
\end{tabular}


Tab. 3 (Fortsetzung)

\begin{tabular}{|c|c|c|c|c|c|c|}
\hline & \multicolumn{2}{|c|}{ Interesse an Politik } & \multicolumn{2}{|c|}{ Wichtigkeit Politik } & \multicolumn{2}{|l|}{ Ehrenamt } \\
\hline & $\begin{array}{l}\text { Modell } 1 \\
\text { (Koeff; } \\
\text { SE) }\end{array}$ & $\begin{array}{l}\text { Modell } 2 \\
\text { (Koeff; } \\
\text { SE) }\end{array}$ & $\begin{array}{l}\text { Modell } 1 \\
\text { (Koeff; } \\
\text { SE) }\end{array}$ & $\begin{array}{l}\text { Modell } 2 \\
\text { (Koeff; } \\
\text { SE) }\end{array}$ & $\begin{array}{l}\text { Modell } 1 \\
\text { (Koeff; } \\
\text { SE) }\end{array}$ & $\begin{array}{l}\text { Modell } 2 \\
\text { (Koeff; } \\
\text { SE) }\end{array}$ \\
\hline $\begin{array}{l}\text { Werteinstellung „Rück- } \\
\text { sicht auf andere neh- } \\
\text { men“ }\end{array}$ & - & $\begin{array}{l}-0,10 \\
(0,08)\end{array}$ & - & $\begin{array}{l}0,11 \\
(0,08)\end{array}$ & - & $\begin{array}{l}0,06 \\
(0,09)\end{array}$ \\
\hline \multicolumn{7}{|l|}{$\mathbf{R}^{2}$} \\
\hline$t_{1}$ & $\begin{array}{l}0,51 * \\
(0,06)\end{array}$ & $\begin{array}{l}0,46^{*} \\
(0,06)\end{array}$ & $\begin{array}{l}0,36 * \\
(0,08)\end{array}$ & $\begin{array}{l}0,36 * \\
(0,07)\end{array}$ & $\begin{array}{l}0,41 * \\
(0,11)\end{array}$ & $\begin{array}{l}0,42 * \\
(0,11)\end{array}$ \\
\hline $\mathrm{t}_{2}$ & $\begin{array}{l}\mathbf{0 , 6 1 *} \\
(\mathbf{0 , 0 3})\end{array}$ & $\begin{array}{l}0,62 * \\
(0,03)\end{array}$ & $\begin{array}{l}0,36 * \\
(0,03)\end{array}$ & $\begin{array}{l}\mathbf{0 , 3 8 *} \\
(\mathbf{0 , 0 3})\end{array}$ & $\begin{array}{l}0,27 * \\
(\mathbf{0 , 0 3})\end{array}$ & $\begin{array}{l}0,27 * \\
(\mathbf{0 , 0 3})\end{array}$ \\
\hline$t_{3}$ & $\begin{array}{l}\mathbf{0 , 7 3 *} \\
(\mathbf{0 , 0 5})\end{array}$ & $\begin{array}{l}0,68 * \\
(0,04)\end{array}$ & $\begin{array}{l}\mathbf{0 , 6 0 *} \\
(\mathbf{0 , 0 7})\end{array}$ & $\begin{array}{l}\mathbf{0 , 5 5 *} \\
(\mathbf{0 , 0 6})\end{array}$ & $\begin{array}{l}\mathbf{0 , 4 1} * \\
(0,07)\end{array}$ & $\begin{array}{l}\mathbf{0 , 4 1} * \\
(0,07)\end{array}$ \\
\hline Intercept & - & $\begin{array}{l}0,37 * \\
(0,06)\end{array}$ & - & $\begin{array}{l}\mathbf{0 , 4 0 *} \\
(0,09)\end{array}$ & - & $\begin{array}{l}0,03 \\
(0,02)\end{array}$ \\
\hline Slope & - & $\begin{array}{l}0,23 \\
(0,14)\end{array}$ & - & $\begin{array}{l}0,24 * \\
(0,12)\end{array}$ & - & $\begin{array}{l}0,05 \\
(0,05)\end{array}$ \\
\hline \multicolumn{7}{|l|}{ Fit Indices } \\
\hline \multicolumn{7}{|l|}{ BIC } \\
\hline $\mathrm{Chi}^{2} ; \mathrm{df} ; p$ & $\begin{array}{l}10,33 ; 1 \\
p<0,05\end{array}$ & $\begin{array}{l}72,55 ; 9 \\
p<0,05\end{array}$ & $\begin{array}{l}18,29 ; 1 \\
p<0,05\end{array}$ & $\begin{array}{l}22,20 ; 9 \\
p<0,05\end{array}$ & $\begin{array}{l}0,60 ; 1 \\
p<0,05\end{array}$ & $\begin{array}{l}13,95 ; 9 ; \\
p=\mathrm{ns}\end{array}$ \\
\hline RMSEA & 0,082 & 0,071 & 0,112 & 0,033 & 0,000 & 0,020 \\
\hline CFI & 0,989 & 0,950 & 0,945 & 0,978 & 1,000 & 0,975 \\
\hline SRMR & 0,041 & 0,044 & 0,052 & 0,023 & 0,008 & 0,031 \\
\hline
\end{tabular}

Berichtet werden geschätzte Stichprobenwerte für Intercept und Slope in der Metrik des Fragebogens sowie standardisierte Koeffizienten, Ein auf dem 5\%-Niveau signifikantes Ergebnis wird durch Hervorhebung (fett) sowie einen * angezeigt

Quelle: DJI-Surveys AID:A I (2009), AID:A II (2014) und AID:A Panel III (2018); eigene Berechnungen ungewichtet

Tab. 3). Eine explorative Analyse zeigt den aufgrund der deskriptiven Ergebnisse zu erwartenden Befund, dass die Annahme einer non-linearen Veränderung eine deutlich bessere Anpassung an die Daten ergibt: Ladungen auf der Slope $t_{1}=0$; $\mathrm{t}_{2}=0,26 ; \mathrm{t}_{3}=2$ (Modellfit: Chi2 $=3,58 ; \mathrm{df}=1 ; p: \mathrm{ns} ; \mathrm{RMSEA}=0,043 ; \mathrm{CFI}=0,992$; SRMR =0,027). Die Wichtigkeit des Lebensbereichs Politik liegt zu Beginn der Studie im mittleren Bereich $(M=3,73$ auf einer Skala von 1-6) und nimmt im Verlauf der Zeit zu. Allerdings setzt die Zunahme erst nach dem zweiten Messzeitpunkt (2014) richtig ein. Folgt man der non-linearen Modellierung, so erhöht sich die Wichtigkeit des Lebensbereichs vom ersten zum zweiten Erhebungszeitpunkt kaum (um 0,12 Skalenpunkte), dann aber deutlich vom zweiten zum dritten Erhebungszeitpunkt. Sowohl im Modell der linearen Modellierung (vgl. Tab. 3), als auch in der non-linearen Modellierung zeigt sich signifikante Varianz im Ausgangswert und in der Veränderung. Ebenfalls in beiden Modellvarianten zeigt sich ein negativer Zusammenhang des Ausgangswerts mit der Veränderung, der dahingehend interpretiert werden kann, dass Personen, die schon zu Beginn dem Lebensbereich Politik eine hohe Wichtigkeit zuschreiben, dessen Bedeutung eher weniger ausbau- 
en. Umgekehrt bedeutet dieses Ergebnis, dass die Gruppe von Personen, die den Lebensbereich Politik zu Beginn für weniger relevant hielten, über die Erhebungen hinweg die stärksten Anstiege auf ihren Antworten verzeichnen.

Das Ehrenamt der befragten Personen startet bei durchschnittlich 0,42 Funktionen im Ehrenamt pro Person und nimmt im Verlauf der Zeit leicht aber konstant und signifikant um ca. 0,04 Funktionen pro Erhebungsintervall ab. Während der durchschnittliche Ausgangswert des ehrenamtlichen Engagements signifikante Varianz zwischen Personen aufweist, variiert die Slope kaum und nicht signifikant. D. h. die Veränderung im Sinne einer Abnahme des Engagements fällt für alle Personen der Stichprobe sehr ähnlich aus. Die Anzahl der Funktionen im Ausgangswert hängt in dem Modell signifikant negativ mit der Veränderung zusammen, d.h. je mehr Funktionen eine Person übernommen hat, desto geringer ist die Abnahme des Engagements über die Zeit. Eine non-lineare Modellierung für IP und Ehrenamt zeigt keine relevante Fit-Verbesserung.

Zur Vorhersage des Ausgangsniveaus und der Veränderung der drei untersuchten Kriterien werden zwei Gruppen von Merkmalen in das Modell aufgenommen: Personenmerkmale der Befragten sowie deren Wertvorstellungen. Die Ergebnisse sind jeweils als Modell 2 in Tab. 3 angetragen. Von besonderer Bedeutung für die politischen Einstellungen sind die allgemeinen Personenmerkmale.

Ein höheres Interesse an Politik wird berichtet von den älteren Personen in der Stichprobe, von Männern (im Vergleich zu Frauen), von Personen ohne Migrationshintergrund und von Personen mit höheren Bildungsabschlüssen. Zudem äußern Personen, denen es im Allgemeinen wichtig ist, „Kritisch zu sein“, höheres Interesse an Politik. Veränderungen am Interesse an Politik sind mit nur zwei Kovariablen signifikant assoziiert: Die allgemeine Zunahme des Interesses ist geringer bei älteren Personen und stärker bei Personen, denen es wichtig ist „Kritisch zu sein“. Insgesamt sagen die in die Analyse aufgenommenen Kovariablen den Ausgangswert signifikant vorher und klären $37 \%$ der Varianz im Intercept auf. Die Veränderung kann nicht ausreichend gut vorhergesagt werden, die Varianzaufklärung beträgt nur $23 \%$.

Die Vorhersage der Wichtigkeit Politik gelingt ähnlich gut. Auch hier ist das Alter prädiktiv für den Ausgangswert. Ältere Personen äußern schon zu Beginn eine höhere Ausprägung auf der Variable Wichtigkeit Politik, ebenso wie Männer (im Vergleich zu Frauen) und Personen mit höherer Bildung. Der Ausgangswert der Wichtigkeit Politik ist zudem auch mit der allgemeinen Wertvorstellung „Kritisch zu sein“" positiv assoziiert. Die Veränderung der Wichtigkeit Politik dagegen zeigt lediglich einen Zusammenhang mit dem Alter der Personen. Die Zunahme der Wichtigkeit Politik ist bei älteren Studienteilnehmerinnen und -teilnehmern geringer ausgeprägt als bei jüngeren. Die ausgewählten Kovariablen können mit $40 \%$ der Varianz im Ausgangswert und $24 \%$ der Varianz der Veränderung die latenten Variablen jeweils signifikant vorhersagen. Der Modellfit ist trotz der linearen Modellierung der Veränderung insgesamt gut.

Schließlich kann das Modell zur Prädiktion des ehrenamtlichen Engagements zwar insgesamt guten Fit aufweisen, jedoch mit den ausgewählten Kovariablen weder den Ausgangsstand noch die Veränderung in bedeutsamer Weise vorhersagen. Lediglich eine höhere Schulbildung und die Relevanz von Pflichtbewusstsein als 
generalisierte Werthaltung sind mit einem höheren ehrenamtlichen Engagement assoziiert.

\subsection{Gegenseitige Beeinflussung der Entwicklung}

Zur Klärung der Forschungsfrage 2 muss das komplexe Bedingungsgefüge der Entwicklung zivilgesellschaftlichen Engagements und politischer Einstellungen nicht nur in der Abhängigkeit von Personenmerkmalen, sondern auch im gegenseitigen Wechselspiel betrachtet werden. In einem autoregressiven Modell mit verzögerten Kreuzbezügen wurden diese modelliert. Abb. 1 illustriert das untersuchte Modell und stellt zugleich die Ergebnisse dar.

Die autoregressiven Pfadkoeffizienten weisen zunächst auf eine relativ hohe intraindividuelle Stabilität der politischen Einstellungen hin sowie auf eine etwas niedrigere, aber dennoch signifikante intraindividuelle Stabilität des ehrenamtlichen Engagements. Das Gesamtmodell kann knapp die Hälfte der Variabilität der politischen Einstellungen $\mathrm{zu} \mathrm{t}_{3}$ und knapp ein Viertel der Variabilität des ehrenamtlichen Engagements zum Abschluss der Studie aufklären. Unter Berücksichtigung der zeitverzögerten Kreuzbezüge sind das ehrenamtliche Engagement und die politischen Einstellungen der Personen zu keinem Zeitpunkt signifikant miteinander korreliert. Während die Personenmerkmale bedeutsame Beziehungen zu den politischen Einstellungen zum Startzeitpunkt zeigen, ist keines davon mit dem ehrenamtlichen Engagement assoziiert. Besonders interessant sind die längsschnittlichen Querbeziehungen zwischen den Konstrukten. Unter Kontrolle des Alters ist in der Stichprobe ein mittleres Alter zu den jeweils drei Erhebungszeitpunkten anzunehmen. Das Ergebnis des Modells zeigt, dass in der frühen Jugend zwischen 11,8 und 16,8 Jahren keine gegenseitigen Bezüge signifikant werden. Während der späteren Jugend und Adoleszenz sind dagegen kleine signifikante Zusammenhänge zu sehen: die Anzahl der im Ehrenamt übernommenen aktiven Funktionen in der mittleren Jugend sagt signifikant die politischen Einstellungen im jungen Erwachsenenalter $(\mathrm{M}=20,3$ Jahre) vorher und die politischen Einstellungen während der mittleren Jugend prädizieren das ehrenamtliche Engagement im jungen Erwachsenenalter. Beide Pfadkoeffizienten sind positiv allerdings recht klein, d.h. früheres höheres Engagement geht später mit etwas stärker ausgeprägten politischen Einstellungen einher und früh stärker ausgeprägte politische Einstellungen gehen später mit etwas höherem Engagement einher.

\section{Diskussion}

Der vorliegende Beitrag untersuchte die Stabilität, Veränderlichkeit sowie Abhängigkeiten zivilgesellschaftlichen Engagements während der Jugend und des jungen Erwachsenenalters. Drei in der Surveyforschung häufiger genutzte Teilbereiche wurden dafür ausgewählt: politisches Interesse und die Wichtigkeit des Lebensbereichs Politik als Indikatoren für die politische Involvierung der befragten Personen sowie die Übernahme von ehrenamtlichen Funktionen und Ämtern in einer Reihe sehr unterschiedlicher Vereine und Verbände. Die empirische Überprüfung der eingangs 
formulierten Forschungsfragen und Hypothesen wurde anhand der Daten der AID:A Studien (AID:A I 2009, AID:A II 2014 und AID:A Panel III 2018) durchgeführt, so dass (nahezu) ein Jahrzehnt intraindividueller Entwicklung nachgezeichnet werden konnte.

Die Modellierung der einfachen Konstruktausprägung und Stabilität bestätigt zum einen die aus früheren Studien abgeleiteten Wachstumsannahmen und kann sie zugleich um Niveauangaben konkretisieren bzw. präzisieren sowie erstmals auch Veränderungen des ehrenamtlichen Engagements über knapp ein Lebensjahrzehnt während der Jugend und Adoleszenz ergänzen. Die in den Hypothesen für Fragestellung 1 angenommene Zunahme des Interesses an Politik sowie der subjektiven Bedeutung des Lebensbereichs Politik wird mit den AID:A Daten bestätigt. Die Studie erweitert damit die von Baumert et al. (2016) sowie von Grob (2006) dokumentierten Befunde der mit dem Alter zunehmenden Involviertheit auf eine deutlich längere Altersspanne von der frühen Jugend bis hinein ins Erwachsenenalter.

Die Zunahme der politischen Involviertheit bewegt sich in AID:A im moderaten Bereich und zeichnet dabei unterschiedliche Verläufe: Während sich die Veränderung des Interesses an Politik am besten linear abbilden lässt, d. h. in beiden Studienabschnitten etwa ähnlich stark ist, nimmt die Wichtigkeit des Lebensbereichs Politik erst im zweiten Studienabschnitt deutlich zu. In Konkurrenz zu den Lebensbereichen Freizeit, Freunde, Familie und Schule bleibt für die jüngeren Jugendlichen Politik randständig. Beginnend mit der mittleren Jugendphase zwischen der zweiten und der dritten Panelwelle wird Politik wichtiger. Während dieses zweiten Abschnitts wird der überwiegende Anteil der Personen volljährig, Damit ergeben sich für die Befragten deutlich mehr Möglichkeiten, sich politisch zu beteiligen, wenn sie dann z. B. für eine politische Partei kandidieren können. Zugleich werden erst in diesem Lebensabschnitt Erwartungen der Gesellschaft hinsichtlich der politischen Partizipation an die Befragten herangetragen, indem sie z. B. zur Wahl aufgefordert werden. Von besonderem Interesse wären daher längsschnittliche Erweiterungen zu Analysen, die es ermöglichen, die unterschiedlichen Altersstufen der Wahlberechtigung in den verschiedenen Bundesländern in ihren Auswirkungen auf das politische Interesse junger Menschen differenziert zu betrachten (s. z. B. Bertelsmann Stiftung 2015), oder Untersuchungen, die Modellversuche wie „Jugendwahlen“ oder ,Probewahlen“ für Jugendliche evaluieren (Hajji et al. 2015). Ein anderer möglicher Mechanismus, der in diesem Zusammenhang genauer untersucht werden sollte, ist die Selbstwahrnehmung der Personen als Jugendliche oder als Erwachsene. Sich politisch einzubringen und sich für Politik zu interessieren, kann für viele allgemein mit dem „Erwachsensein“ assoziiert sein. Gerade aber in den Jahren um die Volljährigkeit und in der frühen Adoleszenz kippt die Einschätzung von Personen von „sich selbst als Jugendliche oder Jugendlicher“ hin zum ,sich als Erwachsene oder Erwachsener" wahrzunehmen (Berngruber in Vorbereitung).

Interessanterweise hängen das Ausgangsniveau der Wichtigkeit von Politik und des ehrenamtlichen Engagements mit der jeweiligen Veränderung zusammen. Für beide sind bei höherem Ausgangsniveau kleinere Veränderungen, d.h. eine geringere Zunahme der Wichtigkeit aber auch eine geringere Abnahme des Engagements zu verzeichnen. Hervorzuheben ist dabei auch das nicht signifikante Ergebnis für Interesse von Politik, dessen Anstieg unabhängig vom Ausgangswert ist. Eine mögliche 
Annahme, dass Personen, die in ihrer frühen Jugend eher gering politisch interessiert sind, auch im späteren Lebensverlauf eine geringere politische Mobilisierung erfahren, bestätigt sich nicht. Egal, ob junge Menschen in ihrer frühen Jugend gering oder stark an Politik interessiert sind, der weitere Anstieg des politischen Interesses im Lebensverlauf ist unabhängig vom Ausgangsniveau. Dies bedeutet aber auch für die durch den 15. Kinder- und Jugendbericht (Deutscher Bundestag 2017a) in Deutschland angestoßene Diskussion zur Stärkung der politischen Bildung für junge Menschen in Schulen und Ausbildungsinstitutionen, dass politische Bildung in allen Bildungsabschnitten ein wichtiger Gegenstand ist, denn wie sehr sich junge Leute später für Politik interessieren ist unabhängig von ihrem frühen Interesse. Der in diesem Beitrag beschriebene Anstieg des politischen Interesses zwischen Jugend- und jungen Erwachsenenalter könnte nicht nur durch intra-individuelle Entwicklungen, sondern auch durch ein zunehmendes Interesse in den entsprechenden Alterskohorten bedingt sein. Ein solcher Anstieg des politischen Interesses bei jungen Leuten in jüngster Zeit wird beispielsweise durch die Zeitreihendaten der Shell Jugendstudien nahegelegt (Schneekloth und Albert 2019). Gille und Kuger (im Druck) konnten anhand der Daten des AID:A Panel III zeigen, dass sich entlang der drei AID:AWellen ein moderater Anstieg des politischen Interesses auf Kohortenebene nachweisen lässt. Aber auch unter Kontrolle der Kohorteneffekte zeigt sich ein Anstieg des politischen Interesses im Lebensverlauf.

Auf größere Unterschiede zwischen den Personen deutet der substanzielle Varianzanteil im Ausgangsniveau sowie in der Veränderung hin. Die Vorhersage des Ausgangsniveaus der drei Konstrukte Interesse an Politik, Wichtigkeit von Politik und ehrenamtliches Engagement zum Startjahr des Panels sowie deren Veränderungen über den 10-Jahres-Zeitraum erweisen sich als ausreichend gut. Unter den berücksichtigten Personenmerkmalen zeigen sich mit dem Forschungsstand konforme aber auch unerwartete, da neue Befunde. Wie erwartet - und auch in den Ergebnissen zur Veränderung direkt bestätigt - ist das Alter jenseits der Studienlaufzeit von besonderer Bedeutung. Zugleich sind jedoch auch das Geschlecht und der Bildungsstand der befragten Personen mit ihrer politischen Involviertheit assoziiert. Detailliertere Analysen zu Geschlechterunterschieden deuten im gleichen Datensatz sogar auf differenzielle Veränderungsmuster zwischen Männern und Frauen hin (Gille und Kuger im Druck): Detailanalysen ermöglichen es, den Niveauunterschied zwischen den Geschlechtern bei ähnlicher Veränderung differenzierter darzustellen als ähnliche starke Zunahmen der Einstellungen bei beiden Geschlechtern zu unterschiedlichen Zeitpunkten.

Ergänzend zu den Personenmerkmalen wurden ausgewählte Wertorientierungen im Modell mitberücksichtig, um annähernd die möglichen Motivationen für zivilgesellschaftliches Engagement abzubilden (Verba et al. 1995). Hier erweist sich für die politische Involvierung vor allem die Werteorientierung ,kritisch sein“ als ein weiterer Erklärungsfaktor. Sich kritisch mit Personen, Dingen oder öffentlichen Ereignissen auseinanderzusetzen hat für junge Menschen auch einen Bezug zur Politik. Dabei ist nicht nur das Grundniveau mit dieser Wertorientierung positiv assoziiert, sondern auch die Zunahme des Interesses an Politik. Bemerkenswert ist zudem der tendenziell signifikante positive Zusammenhang von ehrenamtlichem Engagement und dem Wert „Pflichtbewusstsein“. Die Übernahme von Aufgaben, Ämtern und 
Funktionen in Vereinen und Verbänden verlangt den jungen Leuten eine gewisse Bereitschaft $\mathrm{ab}$, Verantwortung für mehr oder minder stark geregelte Betätigungen oder auch für Personen zu übernehmen, und wird von einer Orientierung an dem Wert Pflichtbewusstsein unterstützt. Die beiden anderen Wertorientierungen sind für dieses Modell irrelevant und scheinen daher eine weniger wichtige Rolle zu spielen. Die häufig vertretene Annahme, dass insbesondere prosoziale Werte ehrenamtliches Engagement bestärken, hat sich daher bisher weder in querschnittlichen Studien bestätigt (vgl. Gille et al. 2014), noch können die vorliegenden längsschnittlichen Ergebnisse sie stützen.

Das Ausmaß des ehrenamtlichen Engagements - hier gemessen über die Anzahl der ausgeübten Funktionen - geht im biographischen Verlauf zurück. Damit lassen sich die in vielen querschnittlichen Studien gemachten Ergebnisse, die das unterschiedliche Engagementausmaß der verschiedenen Statusgruppen junger Menschen beschreiben (Gille 2015; Kausmann et al. 2017) anhand der Ergebnisse des AID:A Panels III als biographische Verläufe bestätigen. Die SchülerInnnen weisen das höchste Engagementniveau auf. Mit Beginn von Ausbildung und Studium geht das Engagement etwas, aber mit dem Eintritt in den Arbeitsmarkt geht die Übernahme ehrenamtlicher Aufgaben deutlich zurück, da sich die zeitlichen Ressourcen hierfür bei den jungen Leuten verknappen. Ein wichtiges Ergebnis für die Engagementförderung stellt der Befund des AID:A Panels III dar, dass hochengagierte Jugendliche (Übernahme mehrerer Ämter) zwar auch im weiteren Lebensverlauf einen Rückgang im Engagement zeigen, diese Abnahme aber deutlich geringer ist als bei Jugendlichen, die beim Panelstart nicht sehr stark engagiert waren. Wenn man junge Menschen zur Übernahme ehrenamtlicher Aufgaben motivieren möchte, so indizieren die Befunde der vorliegenden Studie die Bedeutung eines frühen Starts.

Schließlich konnten mithilfe der vorliegenden Daten erstmals die gegenseitigen Beziehungen zwischen politischer Involviertheit und ehrenamtlichem Engagement dargestellt werden. Die Ergebnisse deuten vor allem auf die hohe Bedeutung der zum Teil gravierenden Brüche und Veränderungen während der Jugend hin. Erst im Erwachsenenalter können sich die theoretisch angenommenen Beziehungen (vgl. Blinkert und Klie 2015) zwischen den beiden Konstruktbereichen darstellen lassen. Auch unter Kontrolle unterschiedlicher Personenmerkmale sind Personen, die eine hohe politische Involviertheit aufweisen auf längere Sicht eher ehrenamtlich engagiert, bringen sich über übernommene Ämter und Funktionen mehr und vielfältiger ein und sind bereit, gesellschaftliche Verantwortung zu übernehmen. Von ähnlicher, zwar geringer aber dennoch signifikanter Relevanz ist der längsschnittliche Zusammenhang zwischen der früheren ehrenamtlichen Beteiligung und den späteren politischen Einstellungen. Zur nachhaltigen Förderung des zivilgesellschaftlichen Engagements in unserer Gesellschaft können daher beide Hebel genutzt werden, die Verantwortungsübernahme im Ehrenamt ebenso wie die Förderung des politischen Interesses oder der subjektiven Bedeutung des Lebensbereichs Politik. Ein besonders wertvolles Ergebnis der Studie ist es darüber hinaus, dass beide Komponenten auch noch von Veränderungen in der mittleren und späten Jugend sowie im frühen Erwachsenenalter profitieren.

Für die hier dargestellten Ergebnisse gilt jedoch einschränkend anzumerken, dass die ehrenamtliche Beteiligung junger Menschen anhand des AID:A-Panels III weder 
in ihrer Komplexität und Vollständigkeit noch differenziert nach unterschiedlichen Engagementfeldern dargestellt werden kann. Die beiden ersten Erhebungswellen von AID:A erfassten das ehrenamtliche Engagement ausschließlich durch die Abfrage der „Übernahme von Ämtern bzw. Funktionen“ in Vereinen, Verbänden und Organisationen. Lediglich die dritte Welle erlaubte darüber hinaus noch zusätzlich die Erfassung freiwilliger bzw. ehrenamtlicher Aktivitäten in weniger stark vorstrukturierten Initiativen oder Projekten. Eine Differenzierung nach verschiedenen Engagementbereichen konnte auch aus Gründen zu geringer Fallzahlen nicht realisiert werden. Schließlich bleibt einschränkend festzuhalten, dass die Datenbasis keine repräsentative Stichprobe darstellt, sondern eher in Richtung höherer Bildungsabschlüsse und eher weniger Personen mit Migrationshintergrund verzerrt ist.

Funding Open Access funding enabled and organized by Projekt DEAL.

Open Access Dieser Artikel wird unter der Creative Commons Namensnennung 4.0 International Lizenz veröffentlicht, welche die Nutzung, Vervielfältigung, Bearbeitung, Verbreitung und Wiedergabe in jeglichem Medium und Format erlaubt, sofern Sie den/die ursprünglichen Autor(en) und die Quelle ordnungsgemäß nennen, einen Link zur Creative Commons Lizenz beifügen und angeben, ob Änderungen vorgenommen wurden.

Die in diesem Artikel enthaltenen Bilder und sonstiges Drittmaterial unterliegen ebenfalls der genannten Creative Commons Lizenz, sofern sich aus der Abbildungslegende nichts anderes ergibt. Sofern das betreffende Material nicht unter der genannten Creative Commons Lizenz steht und die betreffende Handlung nicht nach gesetzlichen Vorschriften erlaubt ist, ist für die oben aufgeführten Weiterverwendungen des Materials die Einwilligung des jeweiligen Rechteinhabers einzuholen.

Weitere Details zur Lizenz entnehmen Sie bitte der Lizenzinformation auf http://creativecommons.org/ licenses/by/4.0/deed.de.

\section{Literatur}

Almond, G. A., \& Verba, S. (1963). The civic culture. Political attitudes and democracy in five nations. Princeton: Princeton University Press.

Baumert, J., Becker, M., Cortina, K., Köller, O., Kropf, M., \& Maaz, K. (2016). Die Entwicklung des politischen Interesses und des Selbstkonzepts der politischen Kompetenz vom Jugend- bis in das Erwachsenenalter. In A. Schippling, C. Grunert \& N. Pfaff (Hrsg.), Kritische Bildungsforschung. Standortbestimmungen und Gegenstandsfelder (S. 323-352). Opladen: Barbara Budrich.

Berngruber, A. (2020). Kind, jugendlich oder erwachsen? Die biographische Selbstwahrnehmung junger Menschen. In S. Kuger \& S. Walper (Hrsg.), Aufwachsen in Deutschland: Alltagswelten 2019. München: DJI.

Bertelsmann Stiftung (2015). Wählen ab 16. Ein Beitrag zur nachhaltigen Steigerung der Wahlbeteiligung. Gütersloh: Bertelsmann.

Blinkert, B., \& Klie, T. (2015). Zivilgesellschaftliches Engagement in Deutschland und Europa. Freiburg: Freiburger Institut für angewandte Sozialwissenschaft e. V.

Dahl, V., \& Abdelzadeh, A. (2017). Self-selection or socialization? The longitudinal relation between civic engagement and political orientations among adolescents. Nonprofit and Voluntary Sector Quarterly. https://doi.org/10.1177/0899764017728363.

Deutscher Bundestag (2017a). Bericht über die Lebenssituation junger Menschen und die Leistungen der Kinder- und Jugendhilfe in Deutschland - 15. Kinder- und Jugendbericht - und Stellungnahme der Bundesregierung (18. Wahlperiode, Drucksache 18/11050). Berlin: BMFSFJ.

Deutscher Bundestag (2017b). Zweiter Engagementbericht. Demografischer Wandel und bürgerschaftliches Engagement: Der Beitrag des Engagements zur lokalen Entwicklung und Stellungnahme der Bundesregierung (18. Wahlperiode, Drucksache 18/11800). Berlin: BMFSFJ.

Gabriel, O.W. (2013). Bürgerbeteiligung in Deutschland. politische bildung, 46(3), 22-36. 
Gaiser, W., \& Gille, M. (2012). Soziale und politische Partizipation. Trends, Differenzierungen, Herausforderungen. In T. Rauschenbach \& W. Bien (Hrsg.), Aufwachsen in Deutschland. AID:A - Der neue DJI-Survey (S. 136-159). Weinheim/Basel: Beltz Juventa.

Gille, M. (2015). Sind junge Menschen heute vereinsmüde? Vereinsaktivitäten und Vereinsengagement von Jugendlichen und jungen Erwachsenen zwischen 2009 (AID:A I) und 2014/15 (AID:A II). In S. Walper, W. Bien \& T. Rauschenbach (Hrsg.), Aufwachsen in Deutschland heute. Erste Befunde aus dem DJI-Survey AID:A 2015 (S. 46-50). München: Deutsches Jugendinstitut e. V. http://www.dji.de/ fileadmin/user_upload/bibs2015/DJI_AIDA_gesamt_v03.pdf.

Gille, M. (2016). Das Verhältnis junger Menschen zur Politik - Politisches Interesse und weitere Aspekte politischer Involvierung bei Jugendlichen und jungen Erwachsenen. In W. Gaiser, S. Hanke \& K. Ott (Hrsg.), Jung - politisch - aktiv?! Politische Einstellungen und politisches Engagement junger Menschen. Ergebnisse der FES-Jugendstudie 2015. Bonn: Dietz.

Gille, M., de Rijke, J., \& Gaiser, W. (2014). Zur Bedeutung ehrenamtlichen Engagements junger Menschen im Kontext gesellschaftlicher und politischer Beteiligung - Entwicklungstendenzen seit 1990 und Bedingungsfaktoren. In M. Löw (Hrsg.), Vielfalt und Zusammenhalt. Verhandlungen des 36. Kongress der Deutschen Gesellschaft für Soziologie in Bochum und Dortmund 2012, Teil 2 (CD-Rom, Sektion 37, Sek37_1.pdf. Beitrag für die Sektion Jugendsoziologie ,Jugend ohne Engagement? Zum sozialen Engagement von Jugendlichen“ auf dem 36. Kongress der Deutschen Soziologie in Bochum und Dortmund 2012).

Gille, M. (2018). Jugend und Politik - ein schwieriges Verhältnis. DJI Impulse 1/2018, 16-19. https://www. dji.de/fileadmin/user_upload/bulletin/d_bull_d/bull119_d/DJI_Impulse119_PolitischeBil dung.pdf. Zugegriffen: 27. Febr. 2020.

Gille, M., \& Kuger, S. (in Druck). Politische Einstellungen von Jugendlichen, jungen Frauen und jungen Männern - Geschlechtsunterschiede in der Einstellungsentwicklung. In A. Buschmeyer \& I. Hofmann-Lun (Hrsg.), Kindheit - Jugend - Geschlecht. Beiträge zu einer empirischen, gendersensiblen Kindheits- und Jugendforschung. München: Deutsches Jugendinstitut.

Grob, U. (2006). Entwicklung und Stabilität von konventionellem politischem Interesse in langfristiger Perspektive. Ergebnisse aus der LifE-Studie. In A. Ittel \& H. Merkens (Hrsg.), Veränderungsmessung und Längsschnittstudien in der Erziehungswissenschaft (S. 61-92). Wiesbaden: Springer.

Hadjar, A., \& Becker, R. (2006). Bildungsexpansion und Wandel des politischen Interesses in Westdeutschland zwischen 1980 und 2002. Politische Vierteljahresschrift, 47(1), 12-34.

Hajji, R., Achour, S., Wilkens, J., \& Schnabel, T. (2015). Praxisforschung zur Juniorwahl. Eine empirische Studie zur Untersuchung der Wirksamkeit des Instruments der Juniorwahl für die politische Bildung am Beispiel von weiterführenden Berliner Schulen. Berlin: Hochschule Magdeburg-Stendal und Freie Universität Berlin.

Kausmann, C., Simonson, J., \& Hameister, N. (2017). Freiwilliges Engagement junger Menschen. Sonderauswertungen des Vierten Deutschen Freiwilligensurveys. Berlin: BMFSFJ.

Klages, H. (1993). Traditionsbruch als Herausforderung. Perspektiven der Wertewandelsgesellschaft. Frankfurt a. M.: Campus.

Reinders, H. (2014). Jugend. Engagement. Politische Sozialisation. Gemeinnützige Tätigkeit und Persönlichkeitsentwicklung in der Adoleszenz. Wiesbaden: Springer VS.

Rippl, S. (2015). Politische Sozialisation. In K. Hurrelmann, U. Bauer, M. Grundmann \& S. Walper (Hrsg.), Handbuch Sozialisationsforschung (7. Aufl., S. 733-752). Weinheim: Beltz.

Schneekloth, U., \& Albert, M. (2019). Jugend und Politik: Demokratieverständnis und politisches Interesse im Spannungsverhältnis von Vielfalt, Toleranz und Populismus. In Shell Deutschland Holding (Hrsg.), Jugend 2019. Eine Generation meldet sich zu Wort. 18. Shell Jugendstudie (S. 47-101). Weinheim: Beltz.

Simonson, J., \& Vogel, C. (2017). Organisationale Struktur des freiwilligen Engagements und Verbesserungsmöglichkeiten der Rahmenbedingungen. In J. Simonson, C. Vogel \& C. Tesch-Römer (Hrsg.), Freiwilliges Engagement in Deutschland - Der Deutsche Freiwilligensurvey 2014 (S. 523-548). Wiesbaden: Springer VS.

Sommer, M., Rucht, D., Haunss, S., \& Zajak, S. (2019). Fridays for Future. Profil, Entstehung und Perspektiven der Protestbewegung in Deutschland (ipb working paper series, 2/2019). https://protestinstitut. eu/wp-content/uploads/2019/08/ipb-working-paper_FFF_final_online.pdf. Zugegriffen: 27. Febr. 2020.

Verba, S., Schlozman, K.L., \& Brady, H.E. (1995). Voice and equality. Civic voluntarism in American politics. Cambridge: Harvard University Press.

Vogel, C., Hagen, C., Simonson, J., \& Tesch-Römer, C. (2017). Freiwilliges Engagement und öffentliche gemeinschaftliche Aktivität. In J. Simonson, C. Vogel \& C. Tesch-Römer (Hrsg.), Freiwilliges Enga- 
gement in Deutschland - Der Deutsche Freiwilligensurvey 2014 (S. 91-152). Wiesbaden: Springer VS.

Walper, S., Bien, W., \& Rauschenbach, T. (Hrsg.). (2015). Aufwachsen in Deutschland heute. Erste Befunde aus dem DJI-Survey AID:A 2015. https://www.dji.de/fileadmin/user_upload/bibs2015/DJI_ AIDA_gesamt_v03.pdf. Zugegriffen: 27. Febr. 2020. 